\title{
Analysis of Energy Harvesting for Green Cognitive Radio Networks
}

\author{
Ali Ö. Ercan*, M. Oğuz Sunay* and Sofie Pollin ${ }^{\dagger}$ \\ *Department of Electrical and Electronics Engineering, Özyeğin University, Istanbul, Turkey 34794 \\ Email: ali.ercan@ozyegin.edu.tr, oguz.sunay@ ozyegin.edu.tr \\ ${ }^{\dagger}$ ESAT-TELEMIC, Katholieke Universiteit Leuven, Leuven, Belgium \\ Email: sofie.pollin@esat.kuleuven.be
}

\begin{abstract}
This paper develops a Markov Chain analysis framework for an RF energy harvesting cognitive radio network where the secondary system users are furnished with finitesized batteries. We consider a network where the only source of energy for the secondary system is the RF signals of the primary system resulting in an inter-play between energy harvesting and transmission for the secondary network. We show that an RF energy harvesting cognitive radio using todays technology is suitable for a delay-tolerant sensor network where the nodes need to transmit sensor output values very sporadically. We observe that the interplay between primary user traffic arrival and departure rates as well as probabilities of missed detection and false alarm for the secondary user detector and available battery size is of importance and that a percentile channel occupation of the primary network on its own is not a sufficient metric.
\end{abstract}

\section{INTRODUCTION}

Machine-to-Machine (M2M) communications, whereby machines around us communicate with one another with limited or no intervention from humans, is becoming increasingly popular for applications ranging from automation for smart grids, transport systems, agricultural systems, industrial production to healthcare, environmental monitoring as well as home networking. It is clear that efficient spectrum usage will become critically important as M2M penetration increases. This increase will also increase the need for green designs for such systems, for both health and environmental reasons. As such, energy-efficient designs for M2M communications will be of primal importance where issues like $\mathrm{CO}_{2}$ emissions, electromagnetic radiations, and spectrum pollution will need to be closely monitored and be factored into the design requirements and solutions utilizing renewable energy sources will likely be favored.

The components of M2M networks need energy sources for operation. It is necessary to provide a way of operating these networks perpetually without requiring external power cables or periodic battery replacement. These requirements, coupled with the necessity for a green design, has prompted energy harvesting technologies be implemented within M2M networks to exploit renewable energy sources [1]. Energy harvesting, which has gained significant interest in both academia and industry recently, refers to the capture of ambient energy, its conversion into a useable form and its storage for future potential use. Various types of energy may be harvested: mechanical energy, electromagnetic energy, thermal energy, solar energy, biological energy etc. Energy harvesting from radio frequency (RF) signals, which is the premise of the system presented in this paper, has its roots in wireless electric power transmission research [2], [3]. Since then, RF energy harvesting has been an active area of research [4]. While the current techniques of energy harvesting from RF signals is not very efficient, it is expected that more advanced technologies will be developed in the near future.

An energy harvesting cognitive radio (CR)-based $\mathrm{M} 2 \mathrm{M}$ communication network design will not only ease the spectrum shortage problem, but also will result in a green design thanks to its complete reliance on renewable energy. In energy harvesting CR systems, there are two co-existing systems: a primary system (PS) that owns the rights to an assigned frequency band, and an energy harvesting secondary system (SS), that attempts to opportunistically access this band whenever it is not used by the PS and whenever it has sufficient energy for transmission. The CR design should ensure that the SS has sufficient resources for the specific application it is designed for while the PS experiences no or minimal impact from the SS presence.

In this paper, we present a Markov Chain-based framework to analyze the feasibility of cognitive radio networks where the only source of energy for SSs is from RF energy harvesting from the PS signals. In general, the operation of energy harvesting CR networks are limited by two fundamental constraints that need to be carefully and simultaneously considered: energy causality constraint which dictates that energy-harvesting nodes may only transmit when they have sufficient energy, and collision constraint that requires an SU to cause minimal, if any, interference to the PU operation [5]. In the RF energy harvesting CR network considered in this paper, there is a trade-off between spectrum availability and energy availability. The analysis presented in this paper highlights how this trade-off plays out for different PS traffic characteristics. We find limits for SS channel utilization when its traffic is infinitely backlogged and when it harvests all of its energy from the PS transmission, which is modeled as an onoff process. We confirm the analytical results through Monte Carlo simulations.

The remainder of the paper is organized as follows. A brief survey on RF-energy harvesting CR networks is given in Section II. The system model and the analysis of the MC of 
the network given in Section III. Results are given in Section IV and conclusions are drawn in Section V.

\section{SuRVEy ON RF ENERGy HARVESTING CR NETwORKS}

The promise of green, spectrally efficient communications has prompted a number of recent research investigating the impact of energy harvesting on cognitive information transfer. The authors in [6] consider a CR network where SS with infinitely backlogged traffic and a simple energy consumption model performs error-free sensing, and finds the optimal allocation of time between information transfer, spectrum sensing and energy harvesting operations. The authors in [7] attempt to maximize the spatial SS throughput subject to SS transmit power and SS density in a given geography using stochastic geometry. The work assumes that an SS user harvests energy from a PS node only if it is sufficiently close. [8] investigates a network where energy-harvesting SSs help increase the PS performance by relaying in return for increased spectrum access opportunities.

Considering the case where the energy to be harvested arrives from a source that is independent from the information transmitting node, [9] finds optimal transmission policies to maximize the throughput and minimize transmission completion time under channel and energy variations when casual channel state information is available at the receiver. [10] finds policies for maximum possible data rates by deciding when and how much of the data to transmit when the energy is harvested from an independent source. [11] considers a CR network and uses a Markov decision process to find optimal and myopic policies for the energy-harvesting, infinitely backlogged SS that specify when to remain idle or conduct sensing and subsequently possibly attempt transmission. Similar to most work summarized above, the energy to be harvested is assumed to arrive from an independent source in this work. The work presented in [12] and [13] develops a partially observable Markov decision process to jointly develop a spectrum sensing policy and sensing detection threshold setting for an energysensing SU to achieve a maximum expected throughput when the energy source is once again independent. The authors in [5] derive the achievable SS throughput when the PS traffic follows a time-homogeneous Markov Chain (MC) and the SS, harvesting energy from an independent source, has infinitely backlogged data.

\section{System Model AND ANALYSIS}

We consider a single channel that is owned by the PS. The PS performs slotted transmission on this channel whenever it has data to send, without consideration of the SS. The slot duration of the PS is $T$. The SS is also assumed to follow a slotted transmission synchronously with the PS, with the same slot duration. At the beginning of each slot, the SS senses the channel for a "sensing period" of duration $T_{s}$. If the channel is found to be empty of PS traffic, and the SS has enough energy, it attempts transmission in the remainder of the slot. If the channel is found busy or there is not enough stored energy, SS performs ambient RF energy harvesting. The coordination of the channel access among users within the PS and SS is assumed to be present, and is out of scope of this paper. Thus we assume the PS and SS as single entities, from this point on.

The PS traffic is assumed to follow an on-off process. That is, given there is no ongoing transmission, the probability that a new packet arrives next slot (head-of-line probability) is $\alpha$. On the other hand, given an ongoing transmission, the probability that no packet arrives next slot is $\beta$. In order to find the limits of the secondary utilization under energy harvesting, SS is assumed to be infinitely backlogged.

Sensing performed by the SS is assumed to be imperfect. At a slot, given an ongoing PS transmission, the event that the SS decides that the channel is empty is called a "missed detection", and happens with probability $p_{\mathrm{MD}}$ independently of other slots. Similarly, given that the channel is empty, the event that the SS decides that the channel is occupied is called a "false alarm", and happens with probability $p_{\mathrm{FA}}$ independently of other slots. The performance of the sensing circuitry of the secondary system determines the values for $p_{\mathrm{MD}}$ and $p_{\mathrm{FA}}$. The achieved utilization levels as a function of the sensing performance as quantified by $p_{\mathrm{MD}}$ and $p_{\mathrm{FA}}$ are explored in the Results Section. As mentioned before, PS transmits at will without consideration of the SS. Thus, an incoming packet is immediately transmitted by the PS. If a collision with the SS transmission occurs due to sensing imperfections, both of the packets of PS and SS are assumed to be lost.

At a given slot, whenever the SS finds the channel to be occupied or it does not have enough energy for transmission, it performs RF energy harvesting. The energy required to transmit for one slot is assumed to be $K$ units, whereas the energy harvested for one slot is assumed to be one unit. The SS is assumed to have a finite battery of size $B$ units. We adopt a discretized energy model, as such, $K$ and $B$ are assumed to be positive integers.

According to the above description, a Markov-Chain (M.C.) model that incorporates the joint state of both the PS and SS is given in Figure 1. The state $(i, j)$ denotes that there is $B-i$ units of energy stored in the SS battery (thus, $i=0$ denotes a full battery) and $j$ pertains to the transmission of PS: at a given slot, if $j=1$, the PS is transmitting and if $j=0$, the channel is empty. For $i \in\{0,1, \ldots, B\}$ and $j \in\{0,1\}$, there are a total of $2(B+1)$ states. The states that are representative of general and corner cases and the corresponding transitions from those states are given in Figure 1. For example, consider the state $(i, 0)$ when the SS has enough energy for transmission, i.e., $i \leq B-K$. Here, the channel is empty. Thus if the SS senses the channel correctly, i.e., does not perform a false alarm, it attempts transmission, its battery depletes by $K$. At the next slot, if a head-of-line packet arrives and the PS starts transmission, the state becomes $(i+K, 1)$. This event happens with probability $\alpha\left(1-p_{\mathrm{FA}}\right)$. If no new packet arrives at PS, the state goes to $(i+K, 0)$. This event happens with probability $(1-\alpha)\left(1-p_{\mathrm{FA}}\right)$. If a false alarm occurs during sensing, SS does not attempt transmission and the battery level remains the 


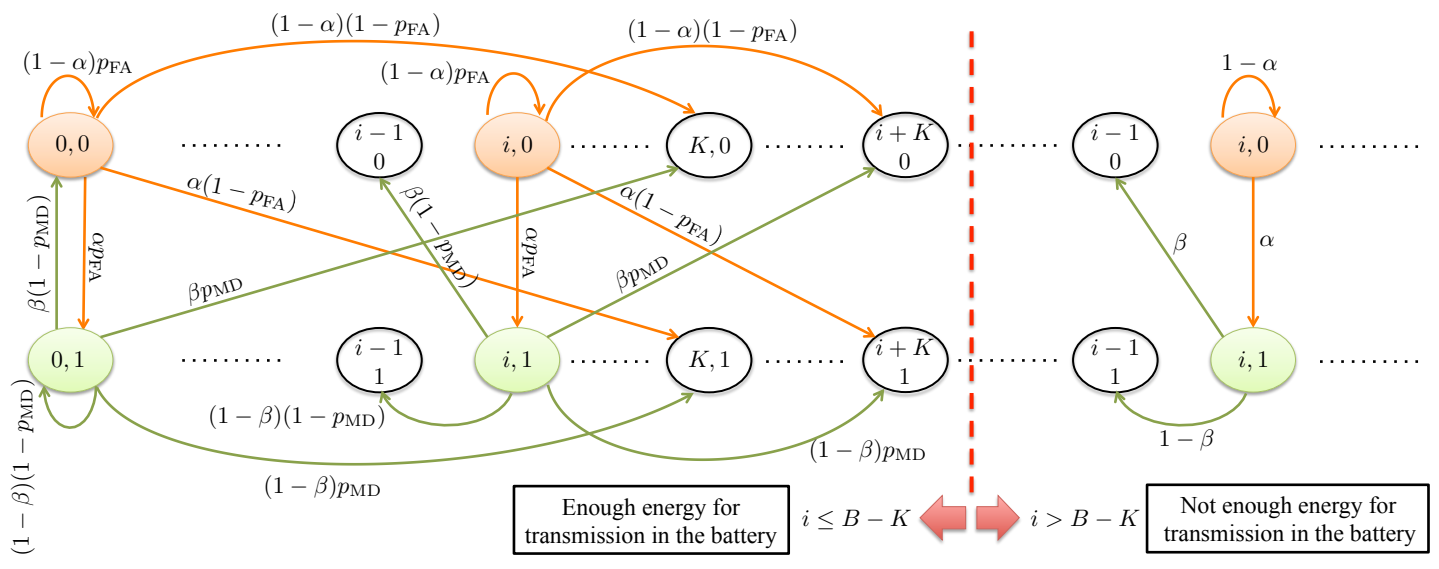

Fig. 1. PS and SS joint Markov Chain.

same. Thus the state transitions to $(i, 0)$ or $(i, 1)$, depending on the packet arrival at the PS. When the SS does not have enough energy for transmission, i.e., $i>B-K$, it does not sense the channel or attempt transmission, thus the state can only go to $(i, 0)$ or $(i, 1)$.

\section{A. PS and SS Utilization Levels}

We define the channel utilization by PS or SS as the average fraction of the time used in successful transmissions. We denote the PS utilization by $U_{P}$ and SS utilization by $U_{S}$. Clearly, $0 \leq U_{S}, U_{P} \leq U_{S}+U_{P} \leq 1$.

Since the joint M.C. described above is finite-state, irreducible and aperiodic, it has a unique steady-state probability mass function (PMF) [14], which can be found numerically as the Perron-Frobenius eigenvector of its state-transition matrix [15]. Given the steady-state PMF, the utilization levels can be found as follows.

On average, the PS transmits $\frac{\alpha}{\alpha+\beta}$ of the slots. Define $\rho$ to be the probability collision at steady-state. Collision occurs at a slot if the PS transmits, SS has enough energy for transmission and performs a missed detection. The probability of this event at steady-state is

$$
\rho=p_{\mathrm{MD}} \sum_{i=0}^{B-K} \Pi_{i, 1},
$$

where $\Pi_{i, j}$ denotes the steady-state probability of the state $(i, j)$. At a fraction $\rho$ of the slots, PS transmissions collide with the SS. Thus, the PS utilization is given by

$$
U_{P}=\frac{\alpha}{\alpha+\beta}-\rho .
$$

Similarly, the SS transmits successfully at a slot if the PS does not transmit, the SS has enough energy for transmission and it does not perform a false alarm, which happens with probability

$$
\gamma=\left(1-p_{\mathrm{FA}}\right) \sum_{i=0}^{B-K} \Pi_{i, 0}
$$

SS utilization is by definition the probability of successful transmission, scaled for the loss in sensing time,

$$
U_{S}=\gamma \frac{T-T_{s}}{T}
$$

\section{REsults}

Using the Markov Chain analysis presented in the previous section, we now investigate the energy harvesting CR network performance for different values of SS sensing circuitry performance as well as PS traffic characteristics and validate the analysis with Monte Carlo simulations.

Following typical link budget values for LTE downlink, we consider a PS system with a transmit power of $46 \mathrm{dBm}$, a transmit antenna gain of $18 \mathrm{dBi}$ and a path loss exponent of 2 [16]. We assume that the SS is about $30 \mathrm{~m}$ away from an eNB. Using Friis equation, we find that the available power for harvesting is approximately equal to $28 \mu \mathrm{W}$ in this case. At the $2 \mathrm{GHz}$ carrier frequency, we assume a harvesting efficiency of $70 \%$ [17], and consider a wireless sensor node with a CC2430 $\mathrm{RF}$ module, the transmission power budget of which is taken to be $26.5 \mathrm{~mW}$ [18], resulting in a transmit power to harvesting power ratio, $K$ of approximately 100 .

A $K$ value of 100 dictates that the utilization of the SS can never be greater than $1 \%$. This is because the SS network is heavily energy-bottlenecked for $K=100$, needing to harvest energy from 100 slots of PS transmissions for every 1 slot of its own transmission. The maximum $1 \% \mathrm{SS}$ utilization can be approximated only when the PS has $99 \%$ channel utilization and the SS sensing is conducted perfectly with $p_{\mathrm{MD}}=p_{\mathrm{FA}}=$ 0 . A $1 \%$ utilization for the SS system limits the use cases for the RF-energy harvesting cognitive radio. Such a system may be useful when the SS is a delay-tolerant sensor network where the nodes need to transmit sensor output values very sporadically.

We conduct performance evaluations using both Markov Chain analysis and Monte Carlo simulations. For the MonteCarlo simulations, we consider 10 runs of PS traffic, composed of 1,000,000 transmission slots and resort the average PS and SS utilization values. In each run, we assume that the SS 


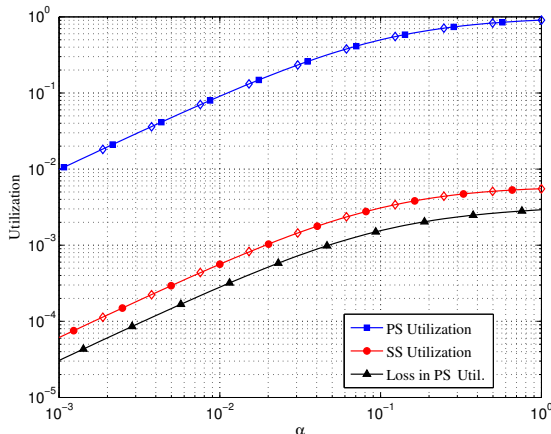

(a)

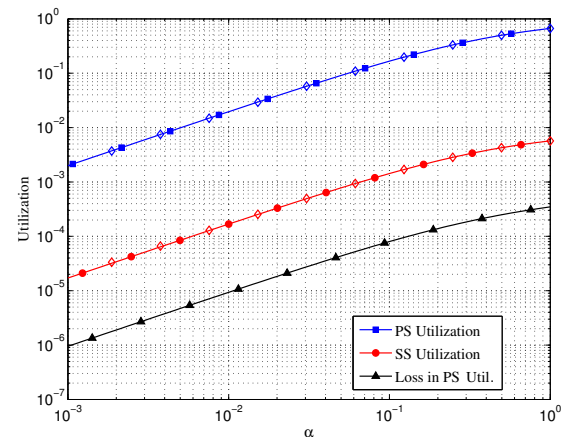

(b)

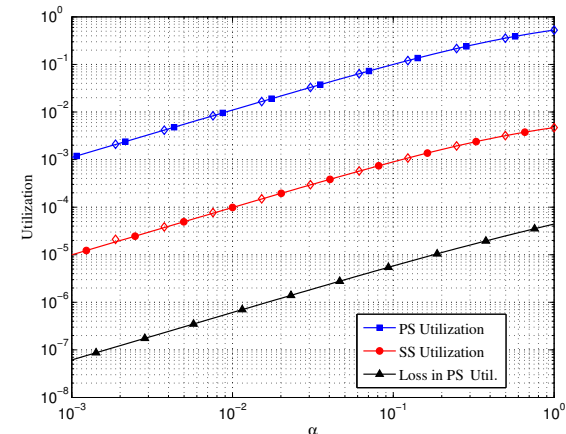

(c)

Fig. 2. Utilization levels as a function of parameter $\alpha$. Here $B=500, p_{\mathrm{MD}}=p_{\mathrm{FA}}=0.05$. (a) $\beta=0.1$, (b) $\beta=0.5$, (c) $\beta=0.9$.

battery of size $B$ starts at level $x$ full, with $x$ uniformly random over $[0, B]$.

We first investigate the performance of the finite-battery energy-harvesting cognitive radio network with $B=500$ and $p_{\mathrm{MD}}=p_{\mathrm{FA}}=0.05$ for different PS traffic characteristics. We let $\beta \in\{0.1,0.5,0.9\}$ and sweep $\alpha$. The results obtained by analysis and Monte Carlo simulations, which are denoted by the empty diamonds, are depicted in Figure 2. We observe a perfect match between the analysis and the Monte-Carlo simulation results, confirming the accuracy of the Markov Chain model presented in the previous section.

Due to the energy-bottlenecked nature of the SS system, we observe in Figure 2 that the SS utilization increases with increasing PS utilization. As the SS utilization increases, not surprisingly, its negative impact on the PS utilization also increases. However, the loss in the PS utilization due to SS presence is always less than the SS utilization. For example, when the PS utilization target is $91 \%$ with $\alpha=1$ and $\beta=0.1$, we observe from Figure 2 (a) that the SS utilization reaches its maximum of 0.0055 and causes a PS utilization loss of only 0.0029 , resulting in an overall observed PS utilization of 0.9062 .

We observe from Figures 2 (a)-(c) that for a given target PS utilization, as $\beta$ increases (i.e., as the PS traffic has shorter bursts), the SS utilization increases. For example, when the PS target utilization is $50 \%$, the corresponding SS utilities for $\beta=0.1,0.5$ and 0.9 become $0.003,0.0042$ and 0.0044 , respectively. This is because, for low $\beta$ values, PS observes long bursts, during which time SU has a chance to fill its battery. Once the battery is full however, if the PS burst is still ongoing, SS cannot transmit nor can harvest. During the corresponding long periods of PS silence, the SS may transmit data for as long as its battery allows. Beyond this point, the channel remains unitized by both networks, causing an effective drop in the SS utilization. With a larger SS battery, this loss could have been prevented. Thus, we conclude that while the overall target PS utilization impacts the overall SS utilization, the PS traffic pattern also has a significant impact when the SS has a finite sized battery. For a given PS utilization level, shorter PS bursts are preferred by the energy bottlenecked SS network.

Next, we investigate the network performance as a function of $p_{\mathrm{MD}}$ and $p_{\mathrm{FA}}$. The results obtained by analysis and Monte Carlo simulations are depicted in Figure 3 (a) and (b), respectively, for $\alpha=\beta=0.1$. Once again, we observe a perfect match between the analysis and Monte-Carlo simulation results. In each graph, when one sensing imperfection is swept, the other is set as 0.05 . We observe that as $p_{\mathrm{MD}}$ increases, the SS utilization decreases. This is because, with increasing $p_{\mathrm{MD}}$, the probability of collision increases. Correspondingly, the impact of the SS network on the PS network increases with increasing $p_{\mathrm{MD}}$. However, since the overall SS utilization is very small when compared with that of the PS, this impact is largely negligible.

We observe that an increase in $p_{\mathrm{FA}}$ has minimal impact on the SS utilization until $p_{\mathrm{FA}}>0.5$. This is because, while a potential SS utilization of $1 \%$ is possible, only $0.3 \%$ is actually realized due to a finite-sized battery, and non-zero $p_{\mathrm{MD}}$ and $p_{\text {FA }}$ values. Then, even when half of the PS idle slots are undetected by the SS for transmission, an attempt to utilize the remaining $25 \%$ of the channel is still bottlenecked by limited available energy. As $p_{\text {FA }}$ gets closer to 1 , we start observing the negative impact of $p_{\mathrm{FA}}$. For values close to 1 , the SS finds only a very small percentage of the actual idle slots useful, resulting in more available energy for transmissions during slots when PS is transmitting, thereby causing collisions and lowering both the SS utilization. For $p_{\mathrm{FA}}=1$, not surprisingly, the SS utilization is equal to zero. As before, since the overall SS utilization is very small when compared with that of the PS, the impact of $p_{\mathrm{FA}}$ on the utilization of the PS is largely negligible.

\section{CONClusion}

In this paper we present a two-dimensional Markov Chain model for an RF energy harvesting cognitive radio network where the primary system (PS) follows a slotted burst traffic model and the secondary system (SS) is synchronized to the PS and has infinitely backlogged traffic. We consider a scenario where the only source of energy for the SS is the RF signals of the PS. The SS has a finite-sized battery to store the energy harvested from the PS transmissions, and utilizes this energy to 


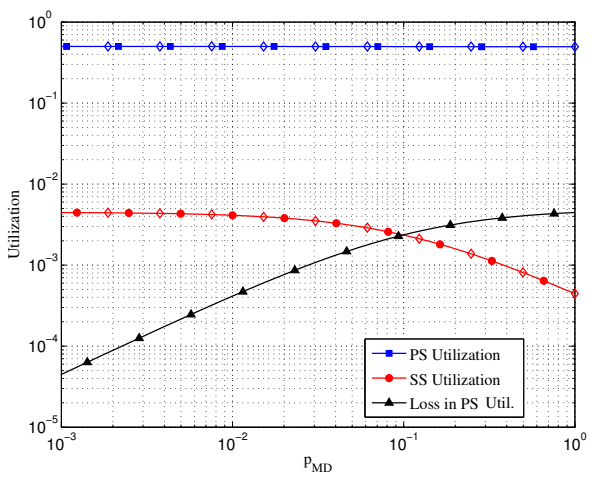

(a)

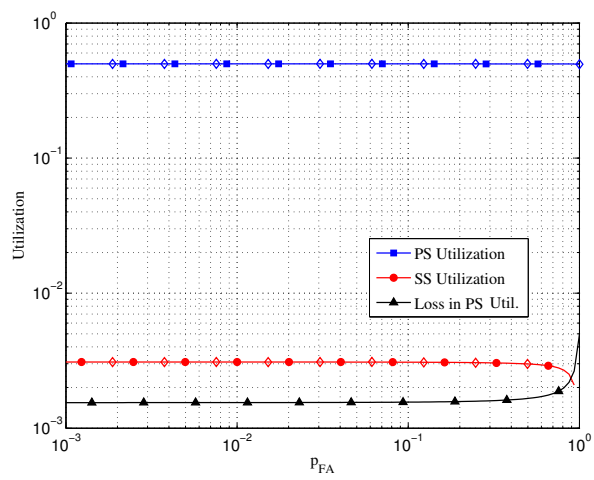

(b)

Fig. 3. Utilization levels as a function of sensing circuitry performance. Here $B=500, \alpha=\beta=0.1$. (a) $p_{\mathrm{MD}}$ is swept, $p_{\mathrm{FA}}=0.05$, (b) $p_{\mathrm{FA}}$ is swept, $p_{\mathrm{MD}}=0.05$.

transmit whenever it senses the channel to be idle. The sensing is considered to be erroneous, where errors are modeled via the probability of false alarm, $p_{\mathrm{FA}}$, and probability of missed detection, $p_{\mathrm{MD}}$. We confirm the accuracy of the Markov Chain model via Monte Carlo simulations.

We use the Markov Chain model and Monte Carlo simulations to assess the PS and SS utilization levels under realistic system parameters. An RF energy harvesting cognitive radio using today's technology is suitable for a delay-tolerant sensor network where the nodes need to transmit sensor output values very sporadically. Specifically, we consider an energybottlenecked SS and conclude that the SS channel utilization is dependent not only on the PS channel utilization but also on its traffic characteristics, described by $\alpha$ and $\beta$ as well as the SS sensing circuit imperfections. Due to the SS energy bottleneck, we observe that the SS utilization increases when the PS utilization also increases. We observe that a PS with short traffic bursts allows the SS to better play out the tradeoff between RF energy harvesting and transmission, resulting in higher SS utilities.

We observe that the $p_{\mathrm{MD}}$ negatively impacts the SS utilization due to an increase in the collision probability with increasing $p_{\mathrm{MD}}$. The corresponding impact on the PS is negligible though, since the SS can only attempt to transmit less than $1 \%$ of the time when the energy harvesting to transmission ratio, $K$ is 100 . We also observe that an increase in $p_{\mathrm{FA}}$ has minimal impact on the SS utilization when $p_{\mathrm{FA}}$ is below a certain value. As $p_{\mathrm{FA}}$ gets closer to 1 however, we start observing that the SS finds only a very small percentage of the actual idle channel slots useful, resulting in more available energy for transmissions during slots when PS is transmitting, thereby causing collisions and lowering the SS utilization. As before, since the overall SS utilization is very small when compared with that of the PS, the impact of $p_{\mathrm{FA}}$ on the utilization of the PS is largely negligible.

\section{REFERENCES}

[1] S. Park, H. Kim, and D. Hong, "Cognitive radio networks with energy harvesting," IEEE Transactions on Wireless Communications, vol. 12, no. 3, pp. 1386-1397, 2013.
[2] N. Shinohara, "Power without wires," IEEE Microwave Magazine, vol. 12, no. 7, pp. S64-S73, December 2011.

[3] W. C. Brown, "The history of power transmission by radio waves," IEEE Transactions on Microwave Theory and Techniques, vol. 32, no. 9, pp. 1230-1242, September 1984.

[4] S. Kitazawa, H. Ban, and K. Kobayashi, "Energy harvesting from ambient RF sources," in Proceedings of IEEE MTT-S, May 2012, pp. $39-42$.

[5] S. Park and D. Hong, "Achievable throughput of energy harvesting cognitive radio networks," IEEE Transactions on Wireless Communications, vol. 13, no. 2, pp. 1010-1022, February 2014.

[6] S. Yin, E. Zhang, L. Yin, and S. Li, "Optimal saving-sensingtransmitting structure in self-powered cognitive radio systems with wireless energy harvesting," in Proceedings of IEEE ICC 2013, June 2013, pp. 2807-2811.

[7] S. Lee, R. Zhang, and K. Huang, "Opportunistic wireless energy harvesting in cognitive radio networks," IEEE Transactions on Wireless Communications, vol. 12, no. 9, pp. 4788-4799, September 2013.

[8] G. Zheng, Z. Ho, E. Jorswieck, and B. Ottersten, "Information and energy cooperation in cognitive radio networks," to appear in IEEE Transactions on Signal Processing, 2014.

[9] O. Ozel, K. Tutuncuoglu, J. Yang, S. Ulukus, and A. Yener, "Transmission with energy harvesting nodes in fading wireless channels: Optimal policies," IEEE Journal on Selected Areas in Communications, vol. 29, no. 8, pp. 1732-1743, September 2011.

[10] V. Sharma, U. Mukherji, V. Joseph, and S. Gupta, "Optimal energy management policies for energy harvesting sensor nodes," IEEE Transactions on Wireless Communications, vol. 9, no. 4, pp. 1326-1336, April 2010.

[11] A. Sultan, "Sensing and transmit energy optimization for an energy harvesting cognitive radio," IEEE Wireless Communications Letters, vol. 1, no. 5, pp. 500-503, October 2012.

[12] S. Park, H. Kim, and D. Hong, "Cognitive radio networks with energy harvesting," IEEE Transactions on Wireless Communications, vol. 12 , no. 3, pp. 1386-1397, March 2013.

[13] S. Park and D. Hong, "Optimal spectrum access for energy harvesting cognitive radio networks," IEEE Transactions on Wireless Communications, vol. 12, no. 12, pp. 6166-6179, December 2013.

[14] A. Leon-Garcia, Probability, Statistics, \& Random Processes for Electrical Engineering, 3rd ed. Prentice Hall, 2007.

[15] C. Mayer, Matrix Analysis and Applied Linear Algebra. Philadelphia: SIAM, 2001.

[16] C. Cox, An Introduction to LTE: LTE, LTE-Advanced, SAE and $4 G$ Mobile Communications. West Sussex, UK: John Wiley \& Sons, 2012.

[17] P. Nintanavongsa, U. Muncuk, D. Lewis, and K. Chowdhury, "Design optimization and implementation for RF energy harvesting circuits," IEEE Journal on Emerging and Selected Topics in Circuits and Systems, vol. 2, no. 1, pp. 24-33, March 2012.

[18] Q. Wang, M. Hempstead, and W. Yang, "A realistic power consumption model for wireless sensor network devices," in Proceedings of IEEE SECON'06, vol. 1, September 2006, pp. 286-295. 